\title{
Power and passion
}

\author{
Masumi Iwai-Takano
}

(C) The Japan Society of Ultrasonics in Medicine 2014

On what occasion does ultrasonography demonstrate its power? When and where can we, as the ones with the knowledge and skills to utilize this diagnostic technique, demonstrate our power?

In daily clinical practice, where normal medical services are available, ultrasonography is just another diagnostic imaging technique. However, ultrasonography is able to tell us what is happening to the patient in front of us. Whether we understand it or not is up to our skills as clinicians. In order to understand the pathophysiology of our patients with information obtained by ultrasonography, in addition to a full knowledge of ultrasonography, it will be necessary to obtain profound insight on their condition, physical findings and laboratory data other than ultrasonographic findings. Being a cardiologist who specializes in ultrasonography, I believe that ultrasonography should not just be applied as a means of diagnostic imaging, but that we should also possess the focus, determination and passion to comprehend pathophysiology from echo images.

On the other hand, how is ultrasonography used in nonordinary clinical practice? I am currently on a bullet train bound for Hiroshima. Sitting next to me is a cardiac surgeon who is sub-specialized in vascular ultrasonography. We both specialize in "ultrasonographic diagnosis" and are also colleagues who carried out leg vein echo screening on deep venous thrombosis (DVT) at shelters during the unprecedented disaster of the Great East Japan Earthquake in March 2011, of which we were also victims.

At the time of the disaster, the Japan Society of Ultrasonics in Medicine sent portable ultrasonic devices to the stricken areas, which we received along with passion and support of the many people who sent them. We could therefore perform DVT screening at shelters and cooperate with central hospitals when necessary. In non-ordinary practice such as a time of disaster, it is our passion and expertise as ultrasonography specialists that make ultrasonography so powerful.

In August 2014, another disaster hit Japan in the form of a deadly landslide that inflicted immense damage to a mountainous region of Hiroshima. I would like to offer my deepest condolences for the people who perished in the horrific event. I would also like to express my great gratitude to the many people working in medical teams established to provide shelter-based treatment for victims. Lessons learnt from the Great East Japan Earthquake have been put to good use. I will be joining one of the teams as an outside medical support staff member, and I will be involved in DVT screening at the shelters, using a portable ultrasonic device borrowed from the Japan Society of Ultrasonics in Medicine. This emergency system of lending ultrasonic devices was established during the Great East Japan Earthquake, thus enabling the passion and support of countless numbers of people, in Japan and overseas, to be both given and received.

By the time this article is published, we will have already celebrated the beginning of a new year. A year in which I hope to enter a new era, spearheaded by the passion and determination of those who perform ultrasonography, demonstrating its power, leading to a better future.

M. Iwai-Takano ( $\square)$

Intensive Care Unit, Fukushima Medical University Hospital,

Fukushima, Japan

e-mail: masumi@fmu.ac.jp 